\title{
Asma e COVID-19: Atualização
}

\section{Asthma and COVID-19: Current evidence}

Data de receção / Received in: 05/05/2020

Data de aceitação / Accepted for publication in: 21/05/2020

\author{
Rev Port Imunoalergologia 2020;28 (2):97-109 \\ Jóni Costa Carvalho', Iolanda Alen Coutinho', Inês Nunes', Ana Luísa Moura', Frederico S. Regateiro',2,3 \\ I Serviço de Imunoalergologia, Centro Hospitalar Universitário de Coimbra \\ ${ }^{2}$ Instituto de Imunologia, Faculdade de Medicina, Universidade de Coimbra \\ ${ }^{3}$ Coimbra Institute for Clinical and Biomedical Reseach (iCBR), Faculdade de Medicina, Universidade de Coimbra
}

\section{RESUMO}

A relação entre asma e a COVID-19 ainda não está esclarecida. Neste artigo, revemos as publicações disponíveis sobre a relação entre estas duas doenças à data de submissão do artigo. Os estudos preliminares não encontraram uma associação definitiva entre a asma e o risco de infeção por SARS-CoV-2 ou de evolução para COVID-19 grave, embora existam diferenças importantes entre estudos realizados em várias localizações. A infeção por SARS-CoV-2 não parece ser causadora de exacerbações de asma, nem alguns sinais característicos de asma, como a sibilância, têm sido descritos nos estudos sobre COVID-19. A suscetibilidade e risco de COVID-19 grave entre os doentes com asma poderá ser diferente dependendo da idade, da gravidade da doença, do grau de controlo, do seu fenótipo ou endótipo, ou do tipo de tratamento implementado. Estudos dirigidos a doentes com asma serão fundamentais para determinar o real impacto da COVID-19 nestes doentes.

Palavras-chave: Asma, COVID-19, SARS-CoV-2, coronavírus, pandemia, comorbilidades, exacerbação por vírus.

\section{ABSTRACT}

The relationship between asthma and COVID-19 is not completely understood yet. We here review the publications on the interplay between these two diseases, as available on the article submission day. The preliminary results did not find a conclusive association between asthma and an increased risk for SARS-CoV-2 infection or severe COVID-19. However, important differences have been found between studies performed in various locations. SARS-CoV-2 infection does not seem to cause 
Jóni Costa Carvalho, Iolanda Alen Coutinho, Inês Nunes, Ana Luísa Moura, Frederico S. Regateiro

asthma exacerbations, and certain typical asthma signs like wheezing were not described in COVID-19 patient series. The susceptibility and risk for COVID-19 in asthma patients may vary according to the patients' age, asthma severity, asthma control, phenotypes/endotypes, or current treatment. Studies analysing COVID-19 specifically in asthma patients will be required to evaluate the real impact of COVID-19 in asthma.

Key-words: Asthma, comorbidities, coronavirus, COVID-19; pandemic, SARS-CoV-2, viral-induced exacerbation.

\section{INTRODUÇÃO}

A doença por coronavírus 2019 (COVID-19) é causada pelo vírus síndrome respiratória aguda grave-coronavírus 2 (SARS-CoV-2). Devido à sua elevada transmissibilidade, a infeção pelo SARS-CoV-2 disseminou-se rapidamente por todo o mundo, tendo sido classificada como pandemia pela OMS apenas três meses após a identificação do primeiro casol.

A infeção por SARS-CoV-2 tem uma apresentação variável, que pode ir de assintomática ou COVID-19 ligeira, com sintomas como febre de curta duração, tosse, anosmia ou ageusia, até COVID-19 grave, com pneumonia atípica, insuficiência respiratória, síndrome de dificuldade respiratória aguda (SDRA), coagulação intravascular disseminada ou morte ${ }^{2}$. O período de incubação varia entre 2 a 14 dias'.

Apesar do seu aparecimento recente, vários fatores de risco para o desenvolvimento de formas graves de COVID-19 têm sido consistentemente identificados em várias populações. Os mais significativos parecem ser a idade avançada e a presença de comorbilidades, entre as quais se incluem doenças respiratórias crónicas, doenças cardíacas, doenças renais, diabetes e hipertensão ${ }^{3}$. Segundo dados preliminares de vários países, a taxa de letalidade por COVID-19 varia entre $2-7 \%$ na população em geral, atingindo os $15-20 \%$ nos doentes com mais de 80 anos $^{3}$. Tendo em consideração que uma proporção dos indivíduos infetados com SARS-CoV-2 não chega a ser diagnosticado (assintomáticos e/ou não testados), as atuais taxas de letalidade poderão estar sobrestimadas.
A COVID-19 afeta predominantemente o trato respiratório ${ }^{2}$. Tendo em conta que as infeções por vírus respiratórios são uma causa frequente de exacerbações de asma, existe a preocupação de que a COVID-19 tenha uma apresentação mais grave em doentes com asma. Desta forma, várias organizações governamentais e outras ligadas à saúde consideraram os doentes com asma como um provável grupo de risco para apresentações graves de COVID-194,5. No entanto, dados epidemiológicos preliminares não parecem confirmar esta suspeita inicial ${ }^{6}$. Neste artigo, revemos a evidência atual sobre o impacto da COVID-19 em doentes com asma, analisando dados sobre as manifestações clínicas nestes doentes e sobre a prevalência da asma nas grandes séries de doentes com COVID-19.

Várias questões se colocam sobre a infeção por SARS-CoV-2 e a COVID-19 em doentes com asma:

I. Quais as características do SARS-CoV-2 que determinam a infeção pulmonar?

2. Poderá a fisiopatologia da asma aumentar o risco de contrair infeção por SARS-CoV-2?

3. Será a infeção por SARS-CoV-2 causadora de exacerbações de asma?

4. Terá o doente asmático um maior risco de desenvolver COVID-19 grave?

5. Existem particularidades da COVID-19 na asma em idade pediátrica?

6. Poderá a medicação usada para controlar a asma, nomeadamente a corticoterapia, influenciar positiva ou negativamente o resultado da infeção porSARS-CoV-2? 
As respostas a estas questões são ainda incompletas, dado o caráter totalmente novo desta infeção e os poucos dados disponíveis nesta fase inicial da pandemia. Analisando os dados atualmente disponíveis, vamos tentar dar resposta a cada uma destas questões separadamente.

\section{VIROLOGIA E PATOGÉNESE DO SARS-COV-2}

O SARS-CoV-2 faz parte da família dos Coronaviridae, uma família de vírus encapsulados de ARN monocatenário de sentido positivo, que causam infeções de origem zoonótica. Conhecem-se cerca de 42 coronavírus que se dividem taxonomicamente em 4 géneros $(\alpha, \beta, \gamma, \delta)$. Os coronavírus humanos ( $\mathrm{HCoV}$ ) pertencem aos géneros alfa e beta, e até ao surgimento do SARS-CoV-2 eram conhecidos apenas seis. Quatro destes vírus, HCoV-NL63, HCoV-229E, HCoV-OC43 e HKUI, causam infeções sazonais que se manifestam, em imunocompetentes, por sintomas ligeiros de rinofaringite. Os outros dois coronavírus até então conhecidos, o SARS-CoV-I e o MERS-CoV, pertencem ao género beta, à semelhança do SARS-CoV-2, e podem provocar síndromes de dificuldade respiratória aguda, tendo sido responsáveis por epidemias recentes (2002 e 2012)'.

O SARS-CoV-2 é transmitido por via respiratória, predominantemente através de gotículas, aerossóis e contacto direto com fómites. Após deposição no epitélio da mucosa oral ou nasal do hospedeiro, o SARS-CoV-2 entra nas células e inicia a sua replicação. $A$ entrada do SARS-CoV-2 nas células ocorre pela ligação da proteína $S$ (spike protein) do vírus à enzima conversora da angiotensina 2 (ECA2), uma aminopeptidase membranar abundantemente expressa no trato respiratório inferior (em particular nas células alveolares tipo II) e no coração. Uma alta afinidade de ligação e uma maior expressão de ECA2 parecem facilitar a infeção por SARS-CoV-21,7.

Fisiologicamente, a ECA2 pulmonar tem uma função protetora em situações inflamatórias ou de stress pulmonar ${ }^{8}$. Uma expressão aumentada desta enzima (upregula- tion) reduz a síntese de alguns mediadores pró-inflamatórios e previne o desenvolvimento de SDRA através da inibição de MAP quinases (enzimas de fosforilação de proteínas associadas a resposta ao stress e apoptose) e das vias de sinalização do NF-kB (um fator de transcrição importante na regulação da resposta imunitária) ${ }^{8}$. Para além disso, a ECA2 foi implicada na redução da inflamação e remodeling das vias aéreas, tendo sido proposta como um alvo terapêutico na asma ${ }^{9}$. A ligação do SARS-CoV-2 à ECA2 reduz a sua expressão membranar (downregulation), levando em consequência à desregulação desta via de proteção pulmonar. Em conjunto com a própria infeção celular pelo vírus, esta desregulação contribui para o desenvolvimento da resposta imunológica e da cascata inflamatória característica da infeção pulmonar pelo SARS-CoV-2 ${ }^{7}$.

Uma meta-análise de vários estudos sobre a expressão do recetor ECA2 nas várias comorbilidades associadas a COVID-19 concluiu que a expressão desta enzima é significativamente maior em doentes com DPOC, fumadores, diabetes ou com hipertensão arterial e pulmonar ${ }^{10}$. Um estudo dirigido a esclarecer a associação entre alergia respiratória e ECA2 avaliou a expressão de ECA2 em esfregaços de epitélio nasal e brônquico (em crianças e adultos, respetivamente) e classificou os indivíduos segundo atopia, com asma/sem asma, e biomarcadores inflamatórios Th2/não Th2 (ex.: FeNO, eosinófilos no sangue periférico). $O$ estudo concluiu que a sensibilização e exposição a aeroalergénios e marcadores inflamatórios Th2 estão associadas a reduções significativas na expressão da ECA2 e que a expressão foi ainda menor nos doentes com o diagnóstico de asma. $\mathrm{Na}$ asma não alérgica não foi encontrada esta redução da expressão de ECA2 ${ }^{\prime \prime}$. Um estudo de coorte analisou a expressão de ECA2 e TMPRSS2 (uma protease também envolvida na entrada do SARS-CoV-2 nas células) em células da expetoração induzida de 330 doentes com asma pertencentes a um programa de asma grave e em 79 participantes saudáveis. O nível de expressão dos dois genes foi semelhante entre os doentes com asma e os indivíduos saudáveis, e entre os doentes 
Jóni Costa Carvalho, lolanda Alen Coutinho, Inês Nunes, Ana Luísa Moura, Frederico S. Regateiro

com asma observou-se que a expressão destas moléculas foi superior em homens, em afro-americanos, e em doentes com diabetes mellitus. A expressão foi menor em doentes com asma sob corticosteroides inalados ${ }^{12}$.

O dano tecidular na COVID-19 é causado, em grande parte, por uma excessiva resposta imunológica à infeção. ${ }^{13}$ A resposta do sistema imunitário ao SARS-CoV-2 apresenta um predomínio Thl com a produção de IFN-gama, IL-I, IL-6, IL-8, IL-I2 e TNF-alfa, o recrutamento de neutrófilos, de monócitos/macrófagos, de linfócitos Thl/ThI7 e a produção de anticorpos específicos ${ }^{13}$.

\section{FISIOPATOLOGIA DA ASMA E SUSCETIBILIDADE PARA A INFEÇÃO POR SARS-COV-2}

As infeções virais do trato respiratório são reconhecidos desencadeantes das exacerbações de asma, contribuindo para cerca de $50-90 \%$ das exacerbações em crianças e para $40-50 \%$ em adultos ${ }^{14}$. Uma resposta antiviral deficitária, a rutura da barreira epitelial brônquica, a inflamação não controlada e a predisposição genética são alguns dos fatores que podem contribuir para uma maior suscetibilidade dos doentes com asma a vírus respiratórios, que podem desencadear exacerbações da doença ${ }^{15}$. A título de exemplo, a indução de apoptose em células epiteliais infetadas por vírus em indivíduos com asma é significativamente menor do que em indivíduos sem asma, e isto pode estar associado à menor expressão de interferões do tipo l e III em indivíduos com asma ${ }^{14-16}$.

O padrão inflamatório Th2, um dos mais prevalentes na asma, foi também associado a maior risco de infeção viral pela correspondente redução de linfócitos $\mathrm{Thl}^{17}$. Em teoria, esta redução da polarização Thl em doentes com asma poderia associar-se a um maior risco de COVID-19 grave, considerando os presumíveis mecanismos de defesa contra o SARS-CoV-2.

Os eosinófilos, células cuja abundância está frequentemente aumentada na via aérea de doentes com asma com endótipos Th2, também poderão ter um papel importante na imunidade a vírus respiratórios (como o rinovírus) $^{18}$, embora outros autores não encontrem suscetibilidade a infeções víricas em doentes com eosinopenia periférica ${ }^{19}$. Dados relativos aos ensaios clínicos dos anticorpos monoclonais anti-IL-5 e anti-IL-5Ralfa (que diminuem o número e função dos eosinófilos em circulação e nos tecidos) também não encontraram aumento do número ou gravidade das infeções víricas nos doentes que receberam o anticorpo monoclonal, quando comparados com os doentes do grupo controlo 20,21 . Um estudo preliminar que incluiu 140 doentes hospitalizados por COVID-19 (em Wuhan) descreve a presença de linfopenia e eosinopenia como alterações presentes na COVID-196, embora não esteja definido se estas alterações são causa ou consequência da infeção.

Em conclusão, embora alguns mecanismos fisiopatológicos possam contribuir para uma maior suscetibilidade dos doentes com asma a infeções virais, não há dados ainda que permitam concluir sobre a suscetibilidade dos doentes com asma à infeção por SARS-CoV-2.

\section{SARS-COV-2 COMO CAUSA DE EXACERBAÇÕES DE ASMA}

O SARS-CoV-2 é o sétimo coronavírus que sabemos ser capaz de infetar humanos. Entre estes sete tipos de coronavírus existem três que podem causar doença grave (SARS-CoV-I, SARS-CoV-2 e MERS-CoV), enquanto outros quatro causam sobretudo doença ligeira, como rinofaringite (HKUI, NL63, OC43 e $229 \mathrm{E})^{1,7}$. $\mathrm{Na}$ ausência de dados concretos sobre o papel do SARS-CoV-2 nas exacerbações de asma, analisamos os dados existentes sobre o papel de outros coronavírus nas exacerbações de asma.

É importante ter em atenção que nem todas as infeções por vírus respiratórios causam exacerbações de asma. Vários estudos demonstram que, para que ocorra 
uma exacerbação de asma durante uma infeção vírica, são necessários cofatores, como a inflamação da via aérea não controlada ou presença de comorbilidades ${ }^{15}$. A título de exemplo, um estudo que avaliou doentes com asma e infeção por rinovírus observou que os doentes que tinham asma mal controlada tiveram exacerbações mais graves do que os que apresentavam asma controlada, alguns dos quais tiveram apenas quadros típicos de rinofaringite sem exacerbação da asma ${ }^{15}$. Comorbilidades que contribuem para as exacerbações por vírus incluem a rinossinusite crónica, a sensibilização a aeroalergénios e o tabagismo ${ }^{22}$. Doentes com níveis elevados de lgE específica a $D$. pteronyssinus e infetados por rinovírus precisaram de tratamento mais prolongado para sibilância aguda, quando comparados com doentes com menores níveis de $\lg \mathrm{E}^{22}$. Um outro estudo, que avaliou 84 crianças hospitalizadas por agudização de asma, concluiu que a associação entre infeção viral e sensibilização alergénica aumentou o risco de admissão hospitalar em 19,4 vezes ${ }^{14}$.

Uma meta-análise de 42 artigos sobre a relação entre infeções respiratórias virais e exacerbações de asma concluiu que os coronavírus "comuns" (causadores de rinofaringites) podem causar exacerbações de asma $^{14}$. No entanto, a revisão sistemática do grupo GA2LEN-DARE considerou que, embora os coronavírus tenham sido identificados em cerca de $\mathrm{I}-4 \%$ das exacerbações (em crianças e adultos), estes ocorriam, na maioria das vezes, como coinfeções, parecendo ter uma baixa ou mesmo nula contribuição para a exacerbação ${ }^{23}$. Os betacoronavírus que causaram epidemias prévias de doença grave (MERS e SARS-CoV-I, o coronavírus mais semelhante ao SARS-CoV-2) não foram causadores de exacerbações da asma ${ }^{24}$.

As manifestações clínicas de COVID-19 mais descritas são a febre, a tosse e a dispneia e em menor frequência mialgias, diarreia, cefaleia e congestão nasal ${ }^{2,25}$. Sinais como sibilância ou outros indicadores de broncospasmo (como hiperinsuflação e tempo expiratório aumentado) foram raramente descritos nos vários estudos disponíveis ao momento, mesmo naqueles que incluem doentes com asma e DPOC (Quadro I). Um estudo que caracterizou os sintomas mais frequentes de 178 doentes hospitalizados com COVID-19 descreveu a presença de sibilância em apenas 12 doentes. Da amostra faziam parte 27 doentes com asma e 17 com DPOC, não tendo sido especificado se a sibilância foi preferencialmente encontrada nos doentes com estas patologias ${ }^{25}$. Outro estudo, que incluiu 16749 hospitalizados, referiu sibilância em cerca de $10 \%$ dos casos, sendo a prevalência total de asma de $14 \%^{26}$. A tosse, dispneia e toracalgia foram os sintomas que mais se relacionaram com a sibilância.

Em conclusão, apesar de ainda não existirem estudos dirigidos a doentes com asma, os dados sugerem que os sinais e sintomas mais característicos de exacerbações de asma não são frequentes nos doentes infetados por SARS-CoV-2. Estes dados são compatíveis com achados à microscopia pulmonar post-mortem por COVID-19, que mostraram um padrão predominante de doença alveolar difusa que habitualmente poupa os bronquíolos e, portanto, sugerem que o SARS-CoV-2 afeta, predominantemente, o parênquima pulmonar sem causar sintomas obstrutivos ${ }^{27}$.

\section{COVID-19 E ASMA COMO COMORBILIDADE}

A mortalidade por SARS-CoV-2 está associada à idade e a comorbilidades ${ }^{28}$. Os estudos relativos aos coronavírus de epidemias anteriores (por MERS e por SARS-CoV-I) não encontraram associação entre a asma e as formas graves das doenças ${ }^{24}$. No entanto, dado o atingimento pulmonar da infeção por SARS-CoV-2 e associação das exacerbações a infeções víricas "em geral", a asma e a DPOC foram algumas das comorbilidades que, antecipadamente, se consideraram fatores de risco para COVID-19 grave 4 . À medida que mais estudos foram sendo publicados, a associação entre DPOC e COVID-19 foi sendo confirmada, mas revelou-se ambígua em relação à asma. No Quadro I, apresentam-se alguns dos estudos disponíveis à data da elaboração do artigo (04 de maio 
Jóni Costa Carvalho, Iolanda Alen Coutinho, Inês Nunes, Ana Luísa Moura, Frederico S. Regateiro

Tabela I. Estudos de doentes com COVID-19 focados nas comorbilidades

\begin{tabular}{|c|c|c|c|c|c|c|}
\hline \multicolumn{7}{|c|}{ Doentes com COVID-19 } \\
\hline \multirow[b]{2}{*}{ Origem } & \multirow[b]{2}{*}{$\begin{array}{l}\text { Número } \\
\text { amostra }\end{array}$} & \multirow[b]{2}{*}{ Contexto } & \multirow[b]{2}{*}{$\begin{array}{l}\text { Idade, } \\
\text { média }\end{array}$} & \multicolumn{3}{|c|}{ Prevalência (\%) } \\
\hline & & & & $\begin{array}{l}\text { Doença } \\
\text { respiratória } \\
\text { crónica }\end{array}$ & Asma & DPOC \\
\hline $\begin{array}{l}\text { China, Wuhan' } \\
\text { (N7 Hospital) }\end{array}$ & 140 & Hospitalizados & 57 & - & $*$ & $\mathrm{I}, 4 \%$ \\
\hline $\begin{array}{l}\text { China, Chinese } C^{32} C^{32} \\
(1 \mathrm{I} / 02 / 20)\end{array}$ & 44672 & $\begin{array}{l}\text { Casos } \\
\text { confirmados }\end{array}$ & 51 & $2,4 \%$ & - & - \\
\hline $\begin{array}{l}\text { China, Wuhan } \\
\text { (Tonji Hospital) }\end{array}$ & 548 & Hospitalizados & 60 & - & $\begin{array}{l}0,9 \% \\
(n=5)\end{array}$ & $\begin{array}{c}3,1 \% \\
(n=17)\end{array}$ \\
\hline $\begin{array}{l}\text { Itália }{ }^{35} \\
\text { (Rede UCl da Lombardia) }\end{array}$ & $|59|$ & $\mathrm{UCl}$ & 63 & - & $*$ & $\begin{array}{c}2 \% \\
(n=42)\end{array}$ \\
\hline $\begin{array}{l}\text { Espanha }^{36} \\
\text { (Hospital Vitoria) }\end{array}$ & 48 & $\mathrm{UCl}$ & 63 & - & * & $\begin{array}{c}38 \% \\
(n=18)\end{array}$ \\
\hline $\begin{array}{l}\text { Alemanha }^{37} \\
\text { (Hospital Aachen) }\end{array}$ & 50 & Hospitalizados & 65 & - & $\begin{array}{l}12 \% \\
(n=6)\end{array}$ & $\begin{array}{l}22 \% \\
(n=11)\end{array}$ \\
\hline $\begin{array}{l}\text { EUA, COVID-Net CDC } 5 \\
(12 / 02 / 20-28 / 03 / 20)\end{array}$ & 7162 & $\begin{array}{l}\text { Casos } \\
\text { confirmados }\end{array}$ & - & $9,2 \%$ & - & - \\
\hline $\begin{array}{l}\text { EUA, COVID-Net CDC } \\
(01 / 03 / 20-30 / 03 / 20)\end{array}$ & 178 & Hospitalizados & 60 & $34,6 \%$ & $17 \%(n=27)$ & $10,7 \%(n=17)$ \\
\hline $\begin{array}{l}\text { EUA, Nova lorque }{ }^{40} \\
\text { (múltiplos hospitais) }\end{array}$ & 5700 & Hospitalizados & 63 & - & $\begin{array}{c}9 \% \\
(n=479)\end{array}$ & $\begin{array}{c}5,4 \% \\
(n=287)\end{array}$ \\
\hline $\begin{array}{l}\text { EUA, Nova lorque }{ }^{42} \\
\text { (Weill Cornell e Lower Manhattan } \\
\text { Hospital) }\end{array}$ & 393 & Hospitalizados & 62 & - & $\begin{array}{l}12,5 \% \\
(n=49)\end{array}$ & $\begin{array}{l}5,1 \% \\
(n=20)\end{array}$ \\
\hline Reino Unido, ISARIC CCP-UK ${ }^{26}$ & 16749 & Hospitalizados & 72 & $\begin{array}{c}19 \% \\
\text { (sem asma) }\end{array}$ & $\begin{array}{c}14 \% \\
(n=2345)\end{array}$ & $*$ \\
\hline $\begin{array}{l}\text { Inglaterra, OpenSAFELY } \\
\text { (GPrNHS-CPNS) }^{39}\end{array}$ & 5683 & $\begin{array}{c}\text { Falecidos } \\
\text { hospitalares }\end{array}$ & - & $\begin{array}{c}22 \% \\
\text { (sem asma) }\end{array}$ & $\begin{array}{c}16 \% \\
(n=911)\end{array}$ & - \\
\hline \multicolumn{7}{|c|}{ Prevalência na população geral } \\
\hline China $^{6}$ & - & - & - & - & $4,2 \%$ & - \\
\hline$E A^{41}$ & - & - & - & - & $7,7 \%$ & - \\
\hline Estado de Nova lorque ${ }^{41}$ & - & - & - & - & $10,1 \%$ & - \\
\hline Reino Unido ${ }^{38}$ & - & - & - & - & $9,6 \%$ & - \\
\hline Portugal $^{34}$ & - & - & - & - & $7,5 \%$ & - \\
\hline
\end{tabular}

CDC: Center for Disease Control. UCI: Unidade de cuidados intensivos. ISARIC CCP-UK: International Severe Acute Respiratory and emerging Infections Consortium Clinical Characterisation Protocol - United Kingdom. GPrNHS-CPNS: General Practice records National Health Service England - COVID-19 inpatient hospital death notifications.

(*): não reportada. (-): dado não disponível. 
de 2020) representativos de vários países e que incluíram na sua análise as comorbilidades presentes em doentes com COVID-19. São também apresentadas as prevalências de asma e DPOC na população geral.

Os primeiros estudos publicados sobre as comorbilidades associadas à COVID-19 foram realizados na China e sugeriram que a asma e a doença alérgica não são fatores de risco para infeção por SARS-CoV-2, estando estas patologias "infranotificadas" nestes doentes ${ }^{6,29}$. Nesses primeiros estudos, também a DPOC não foi considerada um fator de risco, uma vez que a sua prevalência foi de I-3\% entre os doentes com COVID-1929. A título de exemplo, o primeiro estudo publicado sobre as comorbilidades da COVID-19 grave, realizado em 140 doentes hospitalizados, concluiu que a doença alérgica não é fator de risco para COVID-196. Contudo, esta conclusão foi baseada apenas na ausência de doenças alérgicas entre as comorbilidades identificadas. Neste mesmo estudo, o tabagismo foi associado a risco para infeção COVID-19 mais grave ${ }^{30}$.

Uma meta-análise posterior de sete estudos de coorte retrospetivos, relativos a doentes hospitalizados na China, com uma amostra total de 1576 doentes, identificou a doença respiratória crónica como a quarta comorbilidade mais frequente em doentes hospitalizados com COVID-19 (OR=I,5; Cl 95\%: 0,9-2,1\%), demonstrando ainda que as patologias respiratórias se associavam a doença mais grave $(\mathrm{OR}=2,46 ; \mathrm{Cl} 95 \%: 1,76-3,44)^{31}$. Uma outra análise do Chinese Center for Disease Control and Prevention incluiu 44672 casos confirmados de COVID-19 e encontrou uma prevalência de doença respiratória crónica de $2,4 \%$ e uma taxa de mortalidade de $6.3 \%$, superior à dos doentes com hipertensão (6\%) ou com doença oncológica $(5,6 \%)$ e muito superior à dos doentes sem comorbilidades $(0,9 \%)^{32}$. Um estudo mais recente, ambispetivo, em que os doentes hospitalizados por COVID-19 foram contactados posteriormente para informações adicionais, relatou uma prevalência de asma de $0,9 \%$ ( 5 doentes). Destes, $60 \%$ tiveram manifestações graves ${ }^{33}$.
A prevalência de asma na população chinesa é de cerca de 4,2\%, muito inferior à prevalência de DPOC 6 . Nestes estudos, mesmo sobrestimando a proporção de doentes com asma no grupo de "doenças respiratórias crónicas”, a prevalência de asma entre os doentes com COVID-19 foi inferior à da população geral. Esta informação é consistente entre os vários estudos. Contudo, a baixa prevalência de asma entre a população chinesa (menos de metade das prevalências encontradas em Portuga $\left.\right|^{34}$ e em outros países ocidentais), a baixa valorização do doente ou dos médicos, ou a imperfeita notificação poderão ter contribuído para os baixos números encontrados em estudos epidemiológicos generalistas, onde a asma não foi particularmente investigada. Outros fatores de suscetibilidade à infeção, genéticos, ambientais ou relacionados com o tratamento da asma, poderão influenciar também estes resultados epidemiológicos, pelo que a extrapolação para a nossa população deve ser feita com precaução.

Após a propagação global do SARS-CoV-2, estudos epidemiológicos sobre as comorbilidades da COVID-19 foram sendo publicados em outros países. Um estudo italiano em doentes internados em unidades de cuidados intensivos (UCl) por COVID-19 não identificou asma entre as comorbilidades associadas a COVID-19, tendo encontrado uma prevalência de DPOC de $2 \%^{35}$. Resultados semelhantes foram observados em Espanha ${ }^{36}$. Um pequeno estudo realizado na Alemanha incluiu 50 doentes hospitalizados por COVID-19 e identificou asma em 6 doentes (12\%), dos quais 4 evoluíram para SDRA ${ }^{37}$.

Recentemente, foi publicado um estudo do grupo de vigilância de infeções emergentes do Reino Unido, um estudo observacional detalhado com uma das maiores amostras a nível europeu até à data atual ${ }^{26}$. Incluiu 166 hospitais e avaliou um total de 16749 hospitalizados por COVID-19. Cerca de $53 \%$ dos doentes tinham pelo menos uma comorbilidade. A asma foi uma das cinco mais reportadas, com uma prevalência de $14 \%$, superior à prevalência de asma na população geral ${ }^{38}$. A doença pulmonar crónica não asmática foi também 
Jóni Costa Carvalho, lolanda Alen Coutinho, Inês Nunes, Ana Luísa Moura, Frederico S. Regateiro

uma das mais reportadas (19\%). Foram identificadas correlações independentes entre mortalidade hospitalar e as comorbilidades idade avançada, sexo masculino, doença respiratória crónica não asmática, doença cardíaca crónica e doença neurológica crónica, mas não foi identificada associação entre asma e mortalidade. Do total dos doentes internados, faleceram 33\% (idade média de 80 anos, $12 \%$ não tinham nenhuma comorbilidade) e $17 \%$ continuavam a receber cuidados à data da publicação 26 .

Outro estudo recente, de colaboradores da plataforma OpenSAFELY, criada para análise urgente de diversas bases de dados do serviço nacional de saúde inglês (NHS, National Health Service) no contexto da pandemia de COVID-19, associou os dados do registo de saúde eletrónico dos cuidados primários do NHS de Inglaterra com os dados de doentes falecidos em meio hospitalar por COVID-19, cruzando o registo de mais de 17 milhões de doentes $(n=17425445)^{39}$. A sua metodologia permitiu uma análise populacional mais abrangente do que a dos estudos que incluíram apenas doentes hospitalizados. Cerca de 16\% dos doentes tinham asma. Estes foram subdivididos segundo a prescrição (ou não) no último ano de corticosteroide oral (OCS) como indicador de gravidade. Dos doentes com asma a quem foram recentemente prescritos OCS (I,7\% dos doentes com asma $(n=294003))$ faleceram 0,07\% $(n=201)$ por COVID-19, enquanto entre os doentes com asma mas sem prescrição recente de OCS (14,2\% $(n=2479371))$ faleceram $0,03 \%(n=710)$. Após ajuste de variáveis de confundimento (incluindo idade e sexo), o estudo concluiu que a asma se associa a maior mortalidade, quando comparada com os doentes sem asma, sendo o hazard ratio de I,II (Cl 95\%: I,02-I,20) nos doentes com asma sem prescrição recente de OCS e de I,25 (Cl 95\%: I,08-I,44) naqueles com prescrição recente de OCS. Do total de doentes falecidos por COVID-19 (0,03\%, $n=5683), 16 \%$ tinham asma como comorbilidade ${ }^{39}$. Dados provisórios dos EUA (CDC 12 - 28 de março de 2020) reportaram que, de 7162 casos confirmados e com informações sobre as suas comorbilidades, $37,6 \%$ tinham uma ou mais comorbilidades. A doença respiratória crónica foi a segunda mais reportada (9,2\%), estando incluídos neste grupo doentes com asma, DPOC e enfisema ${ }^{5}$. Um relatório seguinte, com informação mais detalhada e relativo a doentes hospitalizados $(n=178)$, descreveu uma prevalência de asma de $12 \%$ e de DPOC de $10,7 \%{ }^{25}$, sendo neste caso a prevalência de asma superior à da população geral entre os hospitalizados. Um estudo realizado no estado de Nova lorque com 5700 doentes hospitalizados encontrou antecedentes de asma em $9 \%$ dos hospita$\operatorname{lizados}^{40}$, uma percentagem superior à prevalência de asma na população do estado $(7,7 \%)^{41}$. Outro estudo, também em Nova lorque, caracterizou 392 doentes hospitalizados por COVID-19 e comparou aqueles que necessitaram de ventilação mecânica invasiva (VMI) com os que não necessitaram, pesquisando as comorbilidades presentes em cada grupo ${ }^{42}$. Foram identificados 49 doentes com asma (I2\% do total). Destes, 17 (35\% dos doentes com asma) precisaram de VMI, uma proporção idêntica à observada em doentes com hipertensão arterial, diabetes e DPOC (35-36\%). Os doentes com asma corresponderam a $13 \%$ de todos os que precisaram de VMI, uma proporção semelhante à de doentes com asma existentes no total da amostra $(12 \%)^{42}$.

De uma forma geral, podemos concluir que os estudos disponíveis analisaram sobretudo doentes com COVID-19 grave, com médias de idades de cerca de 60 anos e o intervalo interquartil em torno dos 50-80 anos, com algum tipo de complicação ou necessidade de internamento, não sendo inteiramente representativos da população de doentes com asma. Apesar desta diferença, a asma não foi associada a maior risco de COVID-19 ou a formas graves de doença na maioria dos estudos disponíveis, não sendo, no entanto, excluída essa hipótese. Um único estudo dos listados no Quadro I concluiu que a asma não é fator de risco para mortalidade entre hospitalizados. Já a DPOC, com prevalências variáveis nos vários estudos, 
foi associada a maior risco de doença grave ${ }^{26}$. Os dados disponíveis são sobretudo de epidemiologia descritiva e a generalidade dos estudos não ajustou a frequência de comorbilidades para variáveis confundidoras. Desta forma, o risco imputável à asma como comorbilidade permanece ainda largamente desconhecido.

Em relação ao tabagismo, um estudo concluiu que os fumadores têm I,4 vezes mais probabilidade de apresentar sintomas graves de COVID-19 (OR=1,4; Cl 95\%: 0,98$-2,00$ ) e têm uma probabilidade 2,4 vezes superior de internamento em $\mathrm{UCl}$, necessidade de $\mathrm{VMI}$ ou morte, em comparação com os não fumadores ( $R R=2,4 ; \mathrm{Cl} 95 \%$ : I,43-4,04) ${ }^{30}$. Em Portugal, cerca de 19\% da população é fumadora e a prevalência de tabagismo nos indivíduos com asma é sobreponível à da população em geral ${ }^{34}$, podendo esta população apresentar um correspondente aumento da gravidade da COVID-19.

\section{COVID-19 E ASMA EM IDADE PEDIÁTRICA}

As crianças são tipicamente mais suscetíveis a complicações respiratórias das infeções por vírus. No entanto, dados obtidos até ao momento demonstram que as crianças apresentam COVID-19 mais ligeira do que os adultos, sendo excecional a ocorrência de mortes por COVID-19 nesta faixa etária ${ }^{43}$. As razões para esta diferença são em larga medida desconhecidas, embora possam estar relacionadas com o menor número de comorbilidades nas crianças ou com mecanismos fisiopatológicos. Alguns dados mostram que a enzima ECA2 poderá ser estrutural e funcionalmente imatura no trato respiratório das crianças ${ }^{44}$ e que, à semelhança do observado em crianças infetadas com SARS-CoV-I, a libertação de citocinas pró-inflamatórias seja menor em crianças do que em adultos ${ }^{45}$.

As manifestações clínicas mais frequentes nas crianças foram febre de curta duração e sintomas de rinofaringite comum $^{43,46}$. Estudos chineses referem que cerca de $2,8 \%$ das crianças com COVID-19 tiveram doença grave (ne- cessidade de oxigenoterapia ou de $\mathrm{UCl}$ ). Nestes estudos não houve referência a sibilância ou broncoespasmo entre os sinais ou sintomas descritos ${ }^{43,46}$. Um estudo chinês mais recente, que caracterizou em detalhe 171 crianças hospitalizadas no hospital de Wuhan, não reportou asma entre as comorbilidades ${ }^{47}$.

Uma análise recentemente realizada pelo CDC dos EUA, de 345 casos pediátricos com infeção por SARS-CoV-2, encontrou antecedentes de doença respiratória crónica (aqui incluída a asma) em $1 \mathrm{I}, 6 \%$ dos doentes, tendo sido a comorbilidade mais reportada ${ }^{48}$. De um estudo de vários hospitais da comunidade de Madrid que envolveu 4I crianças com COVID-19, 5 (I2\%) apresentaram bronquiolite e I crise de asma $(2 \%)^{49}$. Em conclusão, os dados em crianças são ainda muito escassos. A COVID-19 parece ser mais ligeira nestas faixas etárias e não há relatos que associem a asma a maior gravidade da doença.

\section{COVID-19 E CORTICOTERAPIA INALADA E/OU SISTÉMICA PARA O TRATAMENTO DA ASMA}

O uso de corticosteroides sistémicos na COVID-19 foi desencorajado pela OMS após surgirem estudos que sugeriram que o seu uso resultou num aumento da replicação viral, dificultou a eliminação do vírus e exacerbou sintomas ${ }^{50}$. Estudos posteriores demonstraram que para formas graves de COVID-19, desde que os corticosteroides sistémicos sejam utilizados na fase aguda da doença e durante um curto período de tempo, diminuem a necessidade de oxigenoterapia, melhoram os achados radiológicos e diminuem a mortalidade ${ }^{5 !}$.

Uns estudos indicam que os corticosteroides inalados (ICS) aumentam o número de infeções do trato superior, outros referem o contrário ${ }^{51}$. Um estudo de coorte já referido, que analisou a expressão genética de ECA2 e TMPRSS2 em células da expetoração induzida de 330 doentes com asma e em 79 participantes saudáveis, con- 
Jóni Costa Carvalho, Iolanda Alen Coutinho, Inês Nunes, Ana Luísa Moura, Frederico S. Regateiro

cluiu que a expressão de ECA2 e TMPRSS2 foi menor em doentes com asma sob ICS ${ }^{12}$. Estudos in vitro mostraram que a ciclesonida inalada tem atividade antiviral para o SARS-CoV-2 (ensaios clínicos em curso). A budesonida em combinação com glicopirrónio foi também associada a atividade antiviral para outro coronavírus, o HCoV$-229 \mathrm{E}^{52}$. Não existem dados que indiquem se o tratamento com ICS modifica a suscetibilidade ou a gravidade de COVID-19.

A GINA e a SPAIC recomendam que se mantenha o tratamento com corticosteroide inalado, isolado ou em combinação com broncodilatadores de longa ação, para um ótimo controlo da asma ${ }^{53,54}$. O mesmo se aplica aos corticosteroides orais, quer como terapêutica de manutenção, quer como de resgate, sempre que estritamente necessário para o controlo da asma ${ }^{53}$. Relativamente a doentes com asma sob tratamento com biológicos, é aconselhada a continuação do tratamento durante a pandemia da COVID-19. No entanto, um artigo de opinião de peritos da EAACl recomenda a suspensão destes tratamentos em caso de infeção confirmada por SARS$-\mathrm{CoV}-2^{44}$.

\section{CONCLUSÃO}

Neste artigo fazemos uma revisão das publicações atualmente existentes sobre asma e COVID-19. Dada a fase ainda inicial desta pandemia, a evolução da COVID-19 nos doentes com asma permanece largamente desconhecida e, até ao momento, não foi publicado qualquer estudo que avalie, de forma dirigida, o impacto da infeção por SARS-CoV-2 em doentes com asma.

A suscetibilidade e risco de COVID-19 grave entre os doentes com asma poderá ser diferente, dependendo da idade, da gravidade da doença, do grau de controlo, do seu fenótipo ou endótipo, ou do tipo de tratamento implementado. Deste modo, estudos dirigidos a doentes com asma serão fundamentais para determinar o real impacto da COVID-19 nestes doentes.

\section{Lista de Abreviaturas}

ACE2: Enzima conversora de angiotensina 2

ARN: Ácido ribonucleico

$\mathrm{Cl}$ : Confidence interval

COVID-19: Doença causada pelo coronavírus SARS-CoV-2

DPOC: Doença pulmonar obstrutiva crónica

EUA: Estados Unidos da América

FeNO: Fração exalada de óxido nítrico

GA2LEN: Global Asthma and Allergy European Network

DARE: Definition And Risk factors of Exacerbations working group

GINA: Global Initiative for Asthma

ICS: Corticosteroide inalado

IgE: Imunoglobulina E

IL: Interleucina

INF: Interferão

Linfócitos Th: Linfócitos T helper

MAPQ: Proteinas Quinase Activadas por Mitogenos

MERS-CoV: Middle East respiratory syndrome-related coronavirus

NF-KB: Factor nuclear kappa B

NHS: National Health System

OCS: Corticosteroide oral

OMS: Organização Mundial Saúde

OR: Odds ratio

SARS-CoV-I:Síndrome respiratória aguda grave-coronavírus-I SARS-CoV-2: Síndrome respiratória aguda grave-coronavírus-2 SDRA: Síndrome de dificuldade respiratória aguda SPAIC: Sociedade Portuguesa de Imunoalergologia TMPRSS2:Transmembrane protease, serine 2

UCI: Unidade de Cuidados Intensivos

VMI:Ventilação mecânica invasiva

\section{Conflito de interesses}

Este trabalho não recebeu quaisquer apoios financeiros para a sua realização. Frederico S. Regateiro declara ter recebido apoios como palestrante ou consultor científico de AstraZeneca, Novartis, GlaxoSmithKline, TEVA, Sanofi e Lusomedicamenta.

Contacto:

Jóni Costa Carvalho

Serviço de Imunoalergologia

Hospitais da Universidade de Coimbra

Centro Hospitalar e Universitário de Coimbra

Praceta Mota Pinto

3000- 075 Coimbra

Email: joniccarvalho@gmail.com

ORCID: 0000-0002-0444-7745 


\section{REFERÊNCIAS}

I. Gabutti G, D' Anchera E, Sandri F, Savio M, Stefanati A. Coronavirus: Update Related to the Current Outbreak of COVID-19. Infect Dis Ther 2020 Apr; [Epub] Available from: http://link.springer.com/I0.1007/s4012I-020-00295-5.

2. Del Rio C, Malani PN. COVID-19-New Insights on a Rapidly Changing Epidemic. JAMA - J Am Med Assoc 2020;323(14):1339-40 .

3. Onder G, Rezza G, Brusaferro S. Case-fatality rate and characteristics of patients dying in relation to COVID-19 in Italy. JAMA -J Am Med Assoc 2020; 323(18):1775-6.

4. Direcção Geral de Saúde. COVID-19: Fase de mitigação abordagem do doente com suspeita ou infeção por SARS-CoV-2. Norma Orientação Clínica 004/2020. 23/03/2020. Available from: https://covid19.min-saude.pt/normas.

5. Center of Disease Control COVID-19 Response Team. Preliminary estimates of the prevalence of selected underlying health conditions among patients with coronavirus disease 2019. Report United States 12/02/2020-08/03/2020. Available from: https:// www.cdc.gov/mmwr/volumes/69/wr/pdfs/mm69/3e2-H.pdf.

6. Zhang J, Dong X, Cao Y, Yuan Y, Yang Y, Yan, et al. Clinical characteristics of 140 patients infected with SARS-CoV-2 in Wuhan, China. Allergy Eur J Allergy Clin Immunol 2020; 00: I-I2.

7. Yuefei Jin, Haiyan Yang, Wangquan Ji, Weidong Wu, Shuaiyin Chen, Weiguo Zhang, et al. Virology, epidemiology, pathogenesis, and control of COVID-19. Viruses 2020; 12(4), 372.

8. Imai Y, Kuba K, Rao S, Huan Y, Guo F, Guan B, et al. Angiotensin-converting enzyme 2 protects from severe acute lung failure. Nature 2005; 436:112-6.

9. Vaibhav D, Venkateswara A, Pinakin K, Vajir M, Deep P, Kulbhushan T. Activation of angiotensin-converting enzyme 2 (ACE2) attenuates allergic airway inflammation in rat asthma model. Toxicology and applied pharmacology. 2016; 306:17-26.

10. Pinto BGG, Oliveira AER, Singh Y, Jimenez L, Goncalves ANA, Ogava RLT et al. ACE2 Expression is increased in the lungs of patients with comorbidities associated with severe COVID-19. MedRxiv 2020 Mar; [Epub] Available from: http://medrxiv.org/ content/early/2020/03/27/2020.03.21.2004026I.abstract.

II. Jackson DJ, Busse WW, Bacharier LB, Kattan M, O'Connor GT, Wood RA, et al. Association of respiratory allergy, asthma and expression of the SARS-CoV-2 Receptor, ACE2. J Allergy Clin Immunol 2020 Apr; [Epub ahead of print]. Available from: https:// doi.org/10.1016/j.jaci.2020.04.009

12. Peters MC, Sajuthi S, Deford P, Christenson S, Rios CL, Montgomery MT. COVID-19 related genes in sputum cells in asthma: relationship to demographic features and corticosteroids. Am J Respir Crit Care Med 2020 Apr; [Epub] Available from: https:// doi.org/10.1164/rccm.202003-082IOC.
13. Prompetchara E, Ketloy C, Palaga T. Immune responses in COVID-19 and potential vaccines: lessons learned from SARS and MERS epidemic. Asian Pac J Allergy Immunol 2020;38: I-9.

14. Costa LD, Costa PS, Camargos PA. Exacerbation of asthma and airway infection: is the virus the villain? J Pediatr Rio J 2014;90: 542-55.

15. Jartti T, Bønnelykke K, Elenius V, Feleszko W. Role of viruses in asthma. Semin Immunopathol 2020; 42, 6I-74.

16. Adeli M, El-Shareif T, Hendaus MA. Asthma exacerbation related to viral infections: An up to date summary. J Family Med Prim Care 2019;8:2753-9.

17. Lambrecht BN, Hammad H, Fahy JV. The cytokines of asthma. Immunity 2019; 50(4): 975-91.

18. Piñeros YSS, Bal SM, Dijkhuis A, Majoor C, Dierdorp BS, Tamara $\mathrm{D}$, et al. Eosinophils capture viruses, a capacity that is defective in asthma. Allergy Eur J Allergy Clin Immunol 2019;74(10): 1898-909.

19. Gleich GJ, Klion AD, Lee JJ, Weller PF. The consequences of not having eosinophils. Allergy Eur J Allergy Clin Immunol 2013; 68(7):829-35.

20. Busse WW, Bleecker ER, FitzGerald JM, Ferguson GT, Barker P, Sproule $S$ et al. Long-term safety and efficacy of benralizumab in patients with severe, uncontrolled asthma: I-year results from the BORA phase 3 extension trial. Lancet Respir Med 2019; $7(I): 46-59$.

21. Winthrop KL, Mariette X, Silva JT, Benamu E, Calabrese LH, Dumusc A et al. ESCMID Study Group for Infections in Compromised Hosts Consensus Document on the safety of targeted and biological therapies: an infectious diseases perspective (Soluble immune effector molecules [II]: agents targeting interleukins, immunoglobulins and complement factors). Clin Microbiol Infect 2018; 24(2):S2:21-4.

22. Adeli M, El-Shareif T, Hendaus MA. Asthma exacerbation related to viral infections: An up to date summary. J Family Med Prim Care 2019;8:2753-9.

23. Papadopoulos NG, Christodoulou I, Rohde G, Agache I, Almqvist $C$, Bruno A, et al. Viruses and bacteria in acute asthma exacerbations - A GA ${ }^{2}$ LEN-DARE systematic review. Allergy Eur J Allergy Clin Immunol 20II; 66: 458-68.

24. Shaker MS, Oppenheimer J, Grayson M, Stukus D, Hartog N, Hsieh EWY, et al. COVID-19: Pandemic contingency planning for the allergy and immunology clinic. J Allergy Clin Immunol Pract 2020;8(5):1477-88.

25. Garg S, Lindsay K, Whitaker M, O'Halloran A, Cummings C, Holstein R, et al. Hospitalization rates and characteristics of patients hospitalized with laboratory-confirmed coronavirus disease 2019 - COVID-NET, I4 States, March I-30, Morb Mortal Wkly Rep. US Dep Heal Hum Serv Dis Control Prev 2020; 69(15):458-64. 
Jóni Costa Carvalho, Iolanda Alen Coutinho, Inês Nunes, Ana Luísa Moura, Frederico S. Regateiro

26. Docherty AB, Harrison EM, Green CA, Hardwick HE, Pius R, Norman $L$ et al. Features of 16,749 hospitalised UK patients with COVID-19 using the ISARIC WHO Clinical Characterisation Protocol. MedRxiv 2020. [Epub] Available from: https://doi.org/ 10.110I/2020.04.23.20076042.

27. Carsana L, Sonzogni A, Nasr A, Rossi R, Pellegrinelli A, Zerbi P et al. Pulmonary post-mortem findings in a large series of COVID-19 cases from Northern Italy. MedRxiv 2020. [Epub] Available from: https://doi.org/I0.II01/2020.04.19.20054262.

28. Wu Z, McGoogan JM. Characteristics of and important lessons from the coronavirus disease 2019 (COVID-19) outbreak in China: Summary of a report of 72314 cases from the chinese center for disease control and prevention. JAMA - J Am Med Assoc 2020; 323(I3):1239-42.

29. Lupia T, Scabini S, Mornese Pinna S, Di Perri G, De Rosa FG, Corcione S. 2019 novel coronavirus (2019-nCoV) outbreak: A new challenge. J Glob Antimicrob Resist 2020; 21:22-7.

30. Vardavas Cl, Nikitara K. COVID-19 and smoking: A systematic review of the evidence. Tob Induc Dis 2020;18:20.

31. Yang J, Zheng Y, Gou X, Pu K, Chen Z, Guo, Q Zhou Y. Prevalence of comorbidities in the novel Wuhan coronavirus (COVID-19) infection: a systematic review and meta-analysis. Int J Infec Dis 2020;94:91-9.

32. Liu Z, Bing X, Zhi XZ. Epidemiology group of the new coronavirus pneumonia emergency response mechanism of the chinese center for disease control and prevention. Epidemiological characteristics of the new coronavirus pneumonia. Chinese Journal of Epidemiology 2020; 4I(2):145-5I.

33. Li X, Xu S, Yu M, Wang K, Tao Y, Zhou Y, et al. Risk factors for severity and mortality in adult COVID-19 inpatients in Wuhan, J Allergy Clin Immunol 2020; [Epub ahead of print]. Available from: https:// doi.org//0.1016/j.jaci.2020.04.006.

34. Pereira AM, Morais-Almeida M, Sá e Sousa A, Jacinto T, Azevedo LF, Robalo Cordeiro, et al. Environmental tobacco smoke exposure at home and smoking prevalence in the general Portuguese population-The INAsma study. Rev Port Pneumol 2013; 19(3): II4-24.

35. Grasselli G, Zangrillo A, Zanella A, Antonelli M, Cabrini L, Castelli A, et al. Baseline characteristics and outcomes of I59I patients infected with SARS-CoV-2 admitted to ICUs of the Lombardy Region, Italy. JAMA - J Am Med Assoc 2020; 323(I6): I574-8I.

36. Barrasa H, Rello J, Tejada S, Martín A, Balziskueta G, Vinuesa C, et al. SARS-Cov-2 in spanish intensive care: Early experience with 15-day survival in Vitoria. Anaesth Crit Care Pain Med 2020. [Epub ahead of print]. Available from: https://doi.org//0.1016/j. accpm.2020.04.00I.

37. Michael D, Alexander K, Johannes B, Paul B, Bojan H, Christian $B$, et al. Charakteristik von 50 hospitalisierten COVID-19-
-Patienten mit und ohne ARDS. Dtsch Arztebl Int 2020; II7(I6): 27I-8.

38. Mukherjee M, Stoddart A, Gupta RP, Bright IN, Farr A, Heaven $M$, et al. The epidemiology, healthcare and societal burden and costs of asthma in the UK and its member nations: analyses of standalone and linked national databases. BMC Med 2016:14; II3.

39. Williamson E, Walker AJ, Bhaskaran KJ, Bacon S, Bates C, Morton CE, et al. OpenSAFELY: factors associated with COVID-19-related hospital death in the linked electronic health records of 17 million adult NHS patients. MedRxiv 2020. [Epub] Available from: https://doi.org//0.II0I/2020.05.06.20092999.

40. Richardson S, Hirsch JS, Narasimhan M, Crawford JM, McGinn $\mathrm{T}$, Davidson K, et al. Presenting characteristics, comorbidities, and outcomes among 5700 patients hospitalized with COVID-19 in the New York City Area. JAMA - J Am Med Assoc 2020; [Epub ahead of print]. Available from: https://doi.org//0.100I/ jama.2020.6775.

4I. CDC.gov [homepage on the Internet]. Centers for disease control and prevention: Asthma. Most recent national asthma data. (accessed May 2020). Available from: https://www.cdc.gov/asthma/ most_recent_national_asthma_data.htm.

42. Goyal P, Choi JJ, Pinheiro LC, Schenck EJ, Chen R, Jabri A et al. Clinical characteristics of Covid-19 in New York City. N Engl J Med 2020; [Epub] Available from: https://doi.org//0.1056/NEJMc2010419.

43. Rasmussen SA, Thompson LA. Coronavirus disease 2019 and children: What pediatric health care clinicians need to know. JAMA Pediatr. [Epub ahead of print]. Available from: https://jamanetwork.com/journals/jamapediatrics/fullarticle/2764248.

44. Brough HA, Kalayci O, Sediva A, Untersmayr E, Munblit D, Rio $\mathrm{R}$, et al. Managing childhood allergies and immunodeficiencies during respiratory virus epidemics - the 2020 COVID-19 pandemic. Pediatr Allergy Immunol 2020. [Epub ahead of print]. Available from: https://doi.org//0.1III/pai.13262.

45. Ng PC, Lam CWK, Li AM, Wong CK, Cheng FWT, Leung TF et al. Inflammatory cytokine profile in children with severe acute respiratory syndrome. Pediatrics 2004;113(I):7-14.

46. Calvo C, Tagarro A, Otheo E, Epalza C, Grupo de Seguimiento de la Infección por SARS-CoV-2 en la Comunidad de Madrid. Epidemiological update on SARS-CoV-2 infection in Spain. Comments on the management of infection in pediatrics. An Pediatr Barc 2020;92(4):239-40.

47. Lu X, Zhang L, Du H, Zhang J, Li YY, Qu J, et al. SARS-CoV-2 Infection in children. N Engl J Med 2020: 382(17):1663-5.

48. Center of Disease Control COVID-19 Response Team. Coronavirus disease 2019 in children. Report United States 12/02/20-02/04/20. MMWR Morb Mortal Wkly Rep 2020; 69(14):422-6. 
49. Tagarro A, Epalza C, Santos M, Sanz-Santaeufemia F, Otheo E, Moraleda C, et al. Screening and Severity of coronavirus disease 2019 (COVID-19) in children in Madrid, Spain. JAMA Pediatr 2020. [Epub ahead of print]. Available from: https://doi.org//0.100I/jamapediatrics.2020.1346.

50. Russell CD, Millar JE, Baillie JK. Clinical evidence does not support corticosteroid treatment for 2019-nCoV lung injury. Lancet Lond Engl 2020: 395:473-5.

51. Cazeiro C, Silva C, Mayer S, Mariany V, Wainwright CE, Zhang $\mathrm{L}$. Inhaled corticosteroids and respiratory infections in children with asthma: A meta-analysis. Pediatrics 2017;139(3).
52. Halpin DMG, Singh D, Hadfield RM. Inhaled corticosteroids and COVID-19: A systematic review and clinical perspective. Eur Respir J 2020; 55(5) 2001009.

53. Global Initiative for Asthma. Global Strategy for Asthma Management and Prevention, 2020. Available from: https://ginasthma. org/wp-content/uploads/2020/04/GINA-2020-full-report_final_ wms.pdf (accessed April I5 2020).

54. SPAIC.pt [homepage on the Internet]. Sociedade Portuguesa de Alergologia e Imunologia Clínica: Panfleto Asma e Covid - Grupo de Interesse de Asma da SPAIC. [updated April 2020]. Available from: http://www.spaic.pt/noticias/covidl9. 